\title{
British Association of Otolaryngologists Head and Neck Surgeons Summer Meeting, 7-8 September 2005, Edinburgh, Scotland, UK: head and neck abstracts
}

\section{Changing trends in primary management of oral cavity and oropharyngeal squamous cell carcinoma over 20 years at one institution: a review of 712 patients}

A Al-Mutairy, R Kazi, P Rhys-Evans

From the Royal Marsden Hospital, London, UK

\section{Introduction}

We aimed: (1) to analyse changing trends in the primary management of oral cavity and oropharyngeal squamous cell carcinoma over 20 years at one institution; (2) to find the most important predictive factors for survival; and (3) to show the emerging importance of conservation surgery, quality of life issues and multimodal treatment in primary management of these conditions.

\section{Method}

A retrospective review was conducted, assessing patients presenting over the previous 20 years with oral cavity and oropharyngeal squamous cell carcinoma. Of 925 patients, 712 met the inclusion criteria.

\section{Results}

Of the 712 included patients, 490 were male and 222 female (male to female ratio $=2.2: 1$ ). Of the 712 tumours included, 343/712 (48 per cent) were in the oral cavity and $369 / 712$ (52 per cent) were in the oropharynx. Three hundred and ninety patients (55 per cent) underwent radical radiotherapy with computed tomography (representing 139 (36 per cent) of the oral cavity tumours and 251 (64 per cent) of the oropharynx tumours). One hundred and seventy-two patients ( 24 per cent) underwent combined treatment (representing 85 (49 per cent) oral cavity tumours and 87 (51 per cent) oropharynx tumours). A further 150 patients (21 per cent) underwent surgery alone (representing 119 (79 per cent) oral cavity tumours and 31 (21 per cent) oropharynx tumours). From 1984 to 1993 , 69 per cent of patients were treated by radiotherapy alone, 14 per cent by surgery alone and 17 per cent by combined treatment; from 1994 to 2003, these proportions were 44 per cent, 27 per cent and 29 per cent, respectively. There was a significant increase in the five-year survival rate over the two decades, from 64 to 78 per cent for oral cavity tumours $(p=0.02)$ and from 56 to 72 per cent for oropharynx tumours $(p=0.03)$.

\section{Conclusions}

A significant survival advantage was observed for surgical excision compared with other treatment modalities. The changes in the treatment approach to oral cavity and oropharyngeal cancer patients observed in our institution were positively related to increases in the survival rates of these patients.

\section{Effect of thyroid surgery on globus symptoms: a two-year prospective trial}

P Burns*, C Timon* ${ }^{\dagger}$

From the *Royal Victoria Eye and Ear Hospital, St James's Hospital, Dublin, and ${ }^{\dagger}$ Trinity College, Dublin, Ireland

Introduction

The globus sensation is described as a constant feeling of a lump or fullness in the throat, and it may be the presenting symptom of thyroid enlargement. Although both the symptom and the condition are common, there is little in the literature linking them. A two-year prospective study was carried out to ascertain whether there was an association between thyroid surgery and improvement in globus symptoms.

\section{Method}

All patients undergoing thyroid surgery over a two-year period were included in the study. Patients were questioned pre- and post-operatively and their globus symptom scores recorded using a linear quantative index. Size, weight and histological features of thyroid specimens were correlated and statistical analysis performed.

Results

A total of 200 patients were included in the study. Thirty per cent $(n=58)$ were symptomatic for globus preoperatively. Eighty per cent $(n=46)$ of patients' symptoms resolved completely post-operatively $(p \leq 0.0001)$. Patients with histological features of inflammation showed the greatest improvement $(p \leq 0.0001)$. No association between thyroid specimen size and improvement in symptoms was noted.

\section{Conclusions}

Patients who are to undergo thyroid surgery for pathology and who are symptomatic with globus pharyngeus can be reassured that their symptoms will improve or, indeed, resolve.

Pilot study of the expression of carbonic anhydrase IX and glucose transporter-1 in adenoid cystic carcinoma of the head and neck

A Y Isa*, T H Ward ${ }^{\dagger}$, J Homer*, P Oogarah ${ }^{\ddagger}$, N D Slevin ${ }^{\dagger}$ From the ${ }^{*}$ Departments of Surgery, ${ }^{\dagger}$ Clinical Oncology and ${ }^{\ddagger}$ Pathology, Christie Hospital, Manchester, UK

\section{Introduction}

Adenoid cystic carcinomas (ACCs) are tumours with a propensity for perineural spread and distant metastasis. The search for a tumour-specific protein or characteristic that may assist in the development of novel therapies is 
required. Hypoxia in tumours is associated with decreased radiosensitivity and has emerged as a key factor in driving malignant progression via the transcriptional regulation of a number of genes, amongst them vascular endothelial growth factor, glucose transporter-1 (Glut-1) and carbonic anhydrase IX (CAIX). Carbonic anhydrase IX and Glut-1 have been studied as endogenous markers of hypoxia and are associated with poorer outcome in cervical carcinoma and head and neck squamous cell carcinoma.

\section{Method}

The possible role of hypoxia in the chemoresistance and relative radioresistance of ACCs was investigated. Expression of CAIX and Glut-1 was assessed immunohistochemically in 22 cases of ACC.

\section{Results}

Carbonic anhydrase IX expression was low, with a maximum score in only one in four patients. Glucose transporter-1 expression scores were also minimal (two to six) in five patients. There were non-significant trends towards the development of local recurrences, distant metastases and poorer survival in patients with tumours expressing either CAIX or Glut-1; the strongest trend was for the risk of developing local recurrence in patients expressing CAIX $(p=0.0811)$.

\section{Conclusions}

It is unlikely that hypoxia contributes to generalized poor long term outcome of ACCs. Hypoxia modification treatments may still prove useful, although in a minority of patients. The use of CAIX and Glut-1 as prognosticators may be considered, but the key to successful treatment of ACC still remains elusive.

\section{Voice assessment in total laryngectomy patients, using two validated questionnaires}

R Kazi, J de Cordova, A Singh, P Clarke, C Nutting, K Harrington, P Rhys-Evans

From the Head and Neck and Thyroid Unit, Royal Marsden Hospital, London, UK

\section{Introduction}

Measuring the severity of the impact of voice disorders in total laryngectomy patients is difficult. Outcome measurements of voice restoration are important for evaluation of both the patient's voice and their treatment efficacy. This study was undertaken to estimate the voice handicap and its relation to quality of life in total laryngectomy patients, using two different, validated questionnaires.

\section{Methods}

Thirty total laryngectomy patients with no signs of tumour recurrence were identified and enrolled in the study. The amount of disability caused by the voice disorder was assessed using two closed format question and answer tools: the voice-related quality of life measure (VRQOL) (University of Michigan) and the voice handicap index (VHI) (University of Pittsburgh).

\section{Results}

The study included 21 men and nine women, with a median age of 63.9 years (range, 40 to 84 years). The median voice handicap in general was 47.11 (range, 17 to 97). The whole group median for the VRQOL questionnaire was 59.25 (range, 25 to 75 ). We found a strong correlation ( $p<0.001$, Mann-Whitney) between both VRQOL and VHI questionnaire results. The vocal quality of life was higher and the voice handicap lower, although not statistically significantly, in patients with: a history of radiation or chemotherapy; no neck dissection; no reconstruction; and primary puncture (as compared with secondary puncture).

\section{Conclusion}

This study of voice restoration in patients with total laryngectomy found that patients' VRQOL and VHI scores were good, due to recent advances in this field. Correlation between the assessment questionnaires was also good, giving robust measures of voice quality; however, the VHI questionnaire had better item coverage.

\section{Tracheoesophageal compression associated with substernal goitre: correlation of symptoms with cross-sectional imaging findings}

\section{T Mackle, J Meaney, C Timon}

From the St James's Hospital, Dublin, Ireland

\section{Introduction}

To compare subjective tracheoesophageal pressure symptoms, in patients with substernal goitres, with objective cross-sectional radiographic measurements.

\section{Method}

We conducted a prospective study of patients undergoing thyroidectomy. All patients suspected of substernal thyroid extension on clinical examination were questioned in the out-patient clinic regarding compression symptoms and data were recorded in a pre-operative database. Patients were then referred for cross-sectional imaging, which was reviewed by the same consultant radiologist. Results were analysed to assess how closely patients' clinical symptoms correlated with their radiographic and intra-operative findings.

\section{Results}

Of 203 patients evaluated for thyroidectomy over a two-year period, 26 had retrosternal extension confirmed on cross-sectional imaging. Twenty-two (85 per cent) of these had clinical symptoms associated with tracheoesophageal compression. The most common clinical symptom was the sensation of choking or pressure in the throat (77 per cent). Fifteen patients (57.6 per cent) complained of dyspnoea and three patients (11.5 per cent) presented with severe airway symptoms requiring urgent intervention. Patients with airway symptoms including dyspnoea, stridor and coughing were more likely to have significant tracheal narrowing on pre-operative CT imaging. There was also a significant correlation between the perceived severity of lump sensation in the throat and the presence of tracheal and oesophageal displacement and retrotracheal extension. There was no correlation between any clinical symptoms and the degree of substernal extension, goitre size at thoracic inlet or substernal isthmus diameter. Patients' pathology consisted of multinodular goitre (19), Graves disease (one), nodular hyperplasia (two), non-caseating granuloma (one), papillary carcinoma (one) and anaplastic carcinoma (one). One patient required a sternotomy for thyroid excision; all other goitres were excised through a cervical incision.

\section{Conclusions}

In this study, objective pre-operative CT measurements of tracheal and oesophageal displacement, retrotracheal extension and the degree of tracheal compression correlated well with the presence and severity of tracheoesophageal pressure symptoms. However, the patients who 
required airway intervention showed no marked tracheal compression on pre-operative CT imaging.

\section{Investigation of levels of interleukins 10, 12 and 18 in patients with head and neck cancer}

D Mistry* ${ }^{\dagger}$, A Jebreel ${ }^{* \dagger}$, D Loke*, G Dunn*, K Oliver*, J Greenman*, N Stafford*

From the *Hull Royal Infirmary and the ${ }^{\dagger}$ University of Hull, Hull, UK

\section{Introduction}

There has been much interest in the use of cytokines to combat cancer. Interleukins (Il) 10 and 12 are antagonistic molecules which regulate cell-mediated antitumour immunity. Interleukin 18 is synergistic with I1-12 in producing an antitumour immune response. Studies have shown that, in breast and colorectal cancer patients, production of I1-12 is suppressed and that of I1-10 is increased, suggesting an impaired cell-mediated antitumour response. The role of these cytokines is as yet unknown in squamous cell carcinoma (SCC) of the head and neck (which accounts for over 90 per cent of head and neck cancer). The objective of this study was to determine the levels of I1-10, I1-12 and I1-18 in patients with head and neck SCC.

\section{Method}

Blood was obtained from 52 patients with a confirmed diagnosis of primary SCC of the head and neck before they underwent definitive treatment. Blood was also obtained from 20 control patients. Serum levels of I1-10, I1-12 and I1-18 were determined using an enzyme-linked immunosorbent assay.

Results

Of the 52 patients with head and neck SCC, 38 were male and 14 were female. The primary tumour sites were: oral cavity (10); oropharynx (11); larynx (17); hypopharynx (nine); sinonasal (one); and unknown (three). The levels of I1-12 and I1-18 in the serum of patients with SCC of the head and neck were not statistically different to those of the control group ( $p=0.646$ and $p=0.584$ for I1-12 and I1-18, respectively, Mann-Whitney U test). Patients were as likely as controls to have positive serum I1-10 levels ( $p=0.058$, Fisher's exact test).

\section{Conclusions}

We observed no elevation of Il-10 levels or suppression of Il-12 levels in patients with SCC of the head and neck. The Il-18 level was also unchanged in these patients. These findings suggest that the tumour immunology of head and neck SCC is distinct from that of carcinomas at other sites.

\section{A biological model for teaching open tracheostomy}

R Oakley, M Black*, J Tysome ${ }^{\dagger}$, D Mitchell ${ }^{\dagger}$

From the Royal Sussex County Hospital, Brighton, *Guys Hospital, London, and the 'William Harvey Hospital, Ashford, UK

\section{Introduction}

From August 2007 onwards, higher surgical training will aim to produce consultants who are 'emergency-safe' and capable of performing a number of core ENT surgical procedures, from less experienced trainees, in a shorter time frame. Airway management is a key skill in the ENT surgeon's repertoire, which will define the 'emergency-safe' ENT surgeon. The advent of percutaneous techniques has reduced the need for open surgical tracheostomy, with an accompanying reduction in training opportunities. Our objective was to develop a biological model for open surgical tracheostomy, to be used as part of an integrated approach to teaching emergency airway management.

\section{Method}

A biological model based on the sheep's head was developed. The surgical anatomy of the neck was recreated using pig's oesophagus and transposition of the sheep's salivary gland and mylohyoid muscles to mimic the thyroid gland and strap muscles, respectively. Laminate flooring underlay was used to represent the investing layer of cervical fascia. The neck model was used as an aid to learning open surgical tracheostomy.

\section{Conclusions}

This biological model for open surgical tracheostomy provided a practical opportunity for inexperienced surgical trainees to reinforce new skills learnt as part of an airway management course.

\section{Should we apply suction during fine needle cytology of thyroid lesions? A systematic review and meta-analysis}

D D Pothier, A Narula*

From the Gloucestershire Royal Hospital, Gloucester, and *St Mary's Hospital, London, UK

Introduction

In order to determine which method of obtaining samples for thyroid lesion cytology was superior, we compared fine needle aspiration cytology with fine needle sampling without aspiration (FNS). Both methods have advantages and disadvantages, but there is as yet no agreement on which method produces better specimens for cytological diagnosis.

\section{Method}

We undertook a systematic review of the literature and performed a meta-analysis of the results of four crossover trials.

Results

The resulting odds ratio (OR) favoured FNS $(\mathrm{OR}=0.99$, 95 per cent confidence intervals $0.88-1.11$ ) but was not statistically significant. A fifth paper, not included in the meta-analysis, reported results in favour of FNS $(p=0.003)$

\section{Conclusions}

From the meta-analysis, there was no clear evidence that one method was superior to the other. However, taking into consideration all available evidence, it seems that FNS may be easier to perform and may produce better samples than fine needle aspiration cytology.

\section{A comparison of voice analysis between normal, partial and total laryngectomy patients: does partial laryngectomy preserve vocal function?}

A Singh, J de Cordova, R Kazi, H Salama, P Clarke, P Rhys Evans

From the Head and Neck and Thyroid Unit, Royal Marsden Hospital, London, UK

\section{Introduction}

We aimed: (1) to study the acoustic and stroboscopic parameters in patients who had undergone vertical partial laryngectomy, and (2) to compare these parameters 
with those of normal volunteers and total laryngectomy patients.

\section{Method}

We recruited 27 total laryngectomy patients (TL), six vertical partial laryngectomy patients (PL) and 18 normal volunteers (V). Acoustic analysis and videostroboscopy was performed using a computerized speech studio.

\section{Results}

The mean ages in the three groups were $63.9 \pm 10.5$ years (TL), $54.3 \pm 9.5$ years (PL) and $40.9 \pm 13.5$ years $(\mathrm{V})$ The median maximum phonation times were $10.3 \mathrm{sec}$ (TL), $9.4 \mathrm{sec}$ (PL) and $21.8 \mathrm{sec}$ (V). The median numbers of words per minute were 127 (TL), 126 (PL) and $168.5(\mathrm{~V})$. The results for the TL and PL groups showed poorer values and larger variability for all the quantitative measures using connected speech, as compared with our normal volunteers. Glottic closure was incomplete in almost all patients in the PL group. In the TL group, the shape of the neoglottis was variable. The location of the vibrating segment was inconsistent in both groups.

\section{Conclusions}

Voice measurements in the PL group were closer to those in the total laryngectomy group, compared with those in the volunteers. This would suggest that in the PL group voice function, when measured objectively, is significantly worse than that in normal volunteers and approximates better to that in patients, who have had surgical voice restoration.

\section{Release of fractalkine by apoptotic cells: a "find-me" signal for tumour macrophages}

L Truman* ${ }^{\dagger}$, C A Ogden*, R Nibbs, G Graham, C Gregory*

From the Division of Immunology, Infection and Inflammation, University of Glasgow, the *MRC Centre for Inflammation Research, Research Institute for Medical Cell Biology, University of Edinburgh, EH16 4TJ, and ${ }^{\dagger}$ Department of Otolaryngology, Freeman Hospital, High Heaton, Newcastle Upon Tyen, Ne7 7DN.

\section{Introduction}

High rates of apoptosis and frequent tumour-macrophages are associated with a poor prognosis in head and neck neoplasms. Recently, much progress has been made in our understanding of the anti-inflammatory consequences of apoptotic cell clearance by macrophages. However, little is yet known of the mechanisms underlying the preliminary phase of attraction of macrophages toward apoptotic cells.

\section{Method}

Here, we use Burkitt's lymphoma (BL), a tumour that contains frequent apoptotic cells and large numbers of infiltrating macrophages, as a model to study this chemotactic process.

\section{Results}

Results of in vitro transmigration assays show that apoptotic, but not viable, BL cells release chemotactic molecules that attract macrophages. We demonstrate that fractalkine (CX3CL1), which has a 'chemokine on a stalk' structure, that allows it to function both as an adhesion molecule and as chemokine, is expressed on the surface of BL cells and cleaved following the induction of apoptosis. Inhibition of chemokine activity by the viral peptide vMIPII or by anti-fractalkine antibody, suppresses macrophage chemotaxis to apoptotic BL cells.

\section{Conclusions}

We propose that fractalkine release from apoptotic BL cells provides a navigational signal for macrophages in these tumours in order to ensure high efficiency of apoptotic cell clearance. This phagocytic process provides anti-inflammatory cytokines that aid tumour proliferation. We also suggest that release of chemoattractants from apoptotic cells may play a significant role in macrophage recruitment into head and neck cancers. 